\title{
A controlled clinical trial on the effects of exercise on neuropsychiatric disorders and instrumental activities in women with Alzheimer's disease
}

\author{
Efeitos do exercício físico sobre distúrbios neuropsiquiátricos e atividades \\ instrumentais da vida diária em mulheres com doença de Alzheimer: \\ um ensaio clínico controlado
}

\author{
Carla M. C. Nascimento', Camila V. L. Teixeira', Lilian T. B. Gobbi², Sebastião Gobbi', Florindo Stella',
}

\begin{abstract}
Objective: To analyze the influence of a six-month exercise program on neuropsychiatric disorders and on the performance of instrumental activities in elderly patients with Alzheimer's disease (AD). Methods: The study included 20 patients with AD in the mild to moderate stages of the Clinical Dementia Rating (CDR) divided into two groups: the experimental group, composed of 10 women who participated in the six-month exercise program, and the control group, composed of the 10 remaining AD patients who did not take part in an exercise program during the same period. All participants were evaluated using the Mini-Mental State Exam for global cognitive function, the Neuropsychiatric Inventory Questionnaire for neuropsychiatric disorders, and the Pfeffer Functional Activities Questionnaire for the degree of functional impairment. Results: The control group showed functional and neuropsychiatric deterioration in the comparisons between pre- and post-intervention times and between groups. Conclusion: The experimental group showed a propensity for less deterioration in neuropsychiatric disorders and performance of instrumental activities compared to the sedentary group. Trial registration RBR-4qzm6s.
\end{abstract}

Keywords: Alzheimer's disease; physical exercise; physical therapy; neuropsychiatric disorders; instrumental activities; dementia.

\section{Resumo}

\begin{abstract}
Objetivo: Analisar os efeitos de seis meses de intervenção de um programa de atividade física sobre os distúrbios neuropsiquiátricos e o desempenho nas atividades instrumentais da vida diária de idosos com Doença de Alzheimer (DA). Métodos: Foram recrutados 20 pacientes nos estágios entre leve e moderado da DA. Segundo o escore clínico de demência (CDR), foram distribuídos em dois grupos: o grupo treinamento (GT), composto por dez mulheres que participaram de um program de exercícios físicos por um período de seis meses, e o grupo controle (GC), composto por dez outras participantes que não realizaram nenhum tipo de intervenção motora estruturada durante o mesmo período. Todas as participantes foram avaliadas por meio do Miniexame do Estado Mental, para obtenção da caracterização cognitiva; Inventário Neuropsiquiátrico, para identificação dos distúrbios neuropsiquiátricos mais prevalentes e Questionário de Atividades Instrumentais de Pfeffer, para verificação do grau de comprometimento funcional. Resultados: Os participantes do GC mostraram uma deterioração tanto no desempenho das atividades instrumentais quanto na intensificação dos distúrbios neuropsiquiátricos, quando comparados os momentos pré e pós-intervenção. Conclusão: O GT demonstrou uma atenuação da intensificação dos distúrbios neuropsiquiátricos e do desempenho funcional em relação ao grupo sedentário. Ensaio clínico RBR-4qzm6s.
\end{abstract}

Palavras-chave: Doença de Alzheimer; atividade física; fisioterapia; distúrbios neuropsiquiátricos; atividades instrumentais; demência.

Received: 06/01/2011 - Revised: 10/19/2011 - Accepted: 10/29/2011

\footnotetext{
Aging and Physical Activity Laboratory (LAFE), Department of Physical Education, Universidade Estadual Paulista (UNESP), Rio Claro, SP, Brazil

${ }^{2}$ Posture and Locomotion Studies Laboratory (LEPLO), Department of Physical Education, Biosciences Institute, UNESP, Rio Claro, SP, Brazil

${ }^{3}$ Geriatric Psychiatry Clinic, Faculty of Medical Sciences, Universidade Estadual de Campinas (UNICAMP), Campinas, SP, Brazil

Correspondence to: Carla Manuela Crispim Nascimento, Avenida 24-A, 1515, CEP 13506-900, Rio Claro, SP, Brasil, e-mail: carla_unesp@yahoo.com.br
} 


\section{Introduction $: \because$.}

Alzheimer's disease $(\mathrm{AD})$ is the most common type of dementia. It represents more than half the prevalence of dementia in the Brazilian elderly population ${ }^{1}$. The main characteristic of this type of neurodegenerative process is cognitive impairment that can lead to the onset of neuropsychiatric symptoms ${ }^{2}$. This neuropathological process indicates a specific loss of cholinergic neurons in the nucleus basalis of Meynert at the ventral surface of the basal forebrain. This neuropathological process indicates a specific loss of cholinergic neurons in the nucleus basalis of Meynert at the ventral surface of the basal forebrain. The reduction on cholinergic activity could affects and impairs cognitive function and behavior. A reduction in signal intensity in the area corresponding to the anterior lateral nucleus basalis has been significantly correlated with a reduced concentration of grey matter in the bilateral prefrontal cortex, inferior parietal lobule, and cingulate gyrus ${ }^{3}$.

Most studies have focused on the possible benefits of nonpharmacological approaches to cognitive impairment in patients with $\mathrm{AD}^{4-6}$. As the disease progresses, the symptoms include impairment also in behavioral aspects, such as irritability and aggression ${ }^{7}$. Global cognitive impairment reflects a significant presence of neuropsychiatric disorders, and the prevalence of these symptoms is around $60 \%$ in patients with dementia and more than $80 \%$ in institutionalized patients with dementia ${ }^{2,8,9}$. These disturbances increase the suffering of the patient, accelerate the process of the disease, and increase caregiver burden, greatly impairing the quality of life of both patient and caregiver'.

In addition to global cognitive impairment, neuropsychiatric disturbances can lead to a reduction in functionality and difficulty in the performance of activities of daily living, which decrease autonomy and increase the chance of institutionalization of patients with $\mathrm{AD}^{10}$. Matsuda and Saito ${ }^{11}$ showed that impairment in some cognitive domains was directly related to functional impairment in patients with $\mathrm{AD}$. However, neuropsychiatric disorders also showed a direct relationship with the performance of functional activities ${ }^{2,12}$.

In a review, Eggermont et al. ${ }^{13}$ found that exercise can minimize the risk of incapacity associated with disturbances in behavior, sleep, and mood in patients with dementia. Landreville et al. ${ }^{14}$ investigated non-pharmacological approaches to aggressive behavior in institutionalized elderly patients and found only one study based on motor intervention programs. This study was developed by Holmberg ${ }^{15}$ and showed a significant decrease in aggressive behavior in patients with dementia who took part in a motor intervention program when compared with a control group. In a meta-analysis, Heyn, Abreu and Ottenbacher ${ }^{16}$ demonstrated that regular exercise, mainly aerobic exercise, could reduce cognitive decline, with greater impact on the performance of activities of daily living. Stella et al. ${ }^{17}$ and Christofoletti et al. ${ }^{18}$ also found a significant improvement in neuropsychiatric disturbances in demented patients who took part in a long-term regular exercise program compared with sedentary patients.

Therefore, the aim of the present study was to analyze the effects of a multimodal exercise program combined with cognitive stimulation on neuropsychiatric disturbances and performance of functional activities in elderly women with $\mathrm{AD}$. The hypothesis was that the group that completed the proposed intervention would show positive functional outcomes and an attenuation of neuropsychiatric impairment.

\section{Methods : :}

\section{Sample}

Twenty-seven $\mathrm{AD}$ participants were recruited through ads in newspapers, television, and radio and with the assistance of neurologists, gerontologists, psychiatrists, and other physicians who encouraged their patients to participate in the study. The participants were divided into two groups according to their willingness to follow the proposed exercise program: experimental group $(n=15)$ and control group $(n=12)$. All patients were living in a medium-size city (Rio Claro, SP, Brazil). The inclusion criteria were: a) clinical diagnosis of $\mathrm{AD}$ according to the consensus criteria of the National Institute for Neurological and Communicative Disorders and Stroke - Alzheimer's Disease and Related Disorders Association (NINCDS-ADRDA) ${ }^{19}$ and assessment for dementia according to the Diagnostic and Statistical Manual of Mental Disorders (DSM-IV-R) ${ }^{20}$; b) mild to moderate dementia according to Clinical Dementia Rating $(\mathrm{CDR})^{21}$, a scale that assesses the patient's cognitive and functional state ranging from 0 (normal) to 0.5 (questionable dementia), 1 (mild dementia), 2 (moderate dementia), and 3 (severe dementia); c) locomotion ability, preserved vision, and sufficient hearing for compliance with testing procedures (eyeglasses and/or hearing aids were permissible). A physician trained in geriatric psychiatry confirmed the diagnosis to include only patients with mild or moderate AD. Furthermore, he supervised all cognitive and neuropsychiatric assessments while blind to the patient's assignment to the experimental group or the control group.

The sample considered for data analysis included only participants from the experimental group (EG) who attended at least $75 \%$ of the sessions $(n=10$; age $=78.3 \pm 8.19$ years; schooling $=6.3 \pm 3.5$ years; MMSE cognitive status $=13.7 \pm 7.7$ points) and participants from the control group (CG) who completed all assessments over the course of the study $(n=10$; age $=79.4 \pm 6.2$ years; schooling $=5.8 \pm 3.1$ years; MMSE cognitive status $=13.5 \pm 7.3$ points). This group did not participate in any 
type of motor intervention during the study and had not done so for at least one year prior to the research. All assessments for both EG and CG were conducted during the same period.

All patients who fulfilled the inclusion criteria were invited to participate. For this initial analysis, none of the patients declined. Of the 27 participants screened, 2 withdrew from the exercise program for health reasons and complications from the neurodegenerative process (inability to complete the intervention protocol). Of the remaining participants, 3 from the EG did not fulfill the program's minimum attendance requirement (75\% of sessions). Two participants from the CG did not complete the evaluation process due to intensification of disease impairment, pain or other difficulty reported by caregivers.

Both groups comprised patients who had also participated as controls in another study carried out previously in our laboratory. This is a significant limitation of the study, however it is important to consider that the exclusion of some patients because they were randomly assigned to a control group would prevent them from participating in a program that appears to be a good non-pharmacological option for reducing impairment in functional performance. In our country, participation in these interventional studies is often the only opportunity that patients will have to receive this kind of treatment.

\section{Assessment}

All participants were evaluated in two assessments: at baseline and immediately after the end of the program by the same neurologist (single-blinded). The clinical evaluation did not involve any professionals who were participating in the motor intervention program during the period of the study.

The Mini-Mental State Examination (MMSE) ${ }^{22}$ was applied in order to provide a cognitive screening of participants. This instrument is composed of seven domains that evaluate specific cognitive functions: time-space orientation, short-term memory (retention and recall), attention, calculation, and language. The MMSE scores range from 0 to 30 points, and lower values suggest possible cognitive deficits. The reference values proposed for the evaluation of cognitive deficits of this study were suggested by Brucki et al. ${ }^{23}$, whose cutoff for presence of cognitive impairment is schooling: 20 points for illiterates; 25 points for one to four years of schooling; 26.5 points for five to eight years of schooling; 28 points for nine to eleven years of schooling; and 29 points for individuals with higher education (more than eleven years of schooling).

To evaluate the neuropsychiatric disturbances, the Neuropsychiatric Inventory (NPI) ${ }^{24,25}$ was applied. The NPI is a scale that is answered by the caregiver and contains 12 specific domains that consider changes in the patient's behavior: hallucinations, delusions, agitation, depressive symptoms, anxiety, euphoria, apathy/indifference, disinhibition, irritability/lability, aberrant motor behavior, nighttime disturbances, and appetite/eating changes. The scores take into account the frequency and intensity with which the symptoms appear. The final score is given by multiplying the values of frequency and intensity of symptoms. This instrument has good reliability $(\mathrm{ICC}=0.88)$ and good correlation with other validated measures ${ }^{25}$. High scores indicate major neuropsychiatric disorders.

The instrumental activities of daily living were measured by the Pfeffer Functional Activities Questionnaire (Pfeffer) ${ }^{26}$. This instrument is composed of 10 questions with scores ranging from 0 to 3, increasing according to severity. The highest possible score is 30 . Subjects who score higher than 5 are considered to have functional impairment. This measure is associated with cognitive impairment and can help to diagnose dementia ${ }^{27}$. Pfeffer et al. ${ }^{26}$ showed high reliability values (ICC $=0.80$ and 0.97 ) and good criteria validity (sensibility $=0.85$ and specificity=0.81) for this instrument. In a review, Paixão Júnior and Reichenheim ${ }^{28}$ also reported appropriate values of validity and reliability. Furthermore, the Pfeffer has been widely used both in clinical practice and scientific research ${ }^{29}$.

The present research was approved by the Ethics Committee of Universidade Estadual Paulista (UNESP), Rio Claro, SP, Brazil (protocol number 0846). The participants' legal guardians signed the consent form.

\section{Intervention protocol}

All participants were assisted by an interdisciplinary program that consisted of cognitive stimulation therapy, occupational therapy, and physical education. The intervention was held three times a week in one-hour sessions and included aerobics (moderate intensity over long duration) and different types of activities that simultaneously benefited other components of functional capacity, such as flexibility (stretching), muscular resistance (specific exercises for large muscle groups and with series of 15/20 repetitions according to the volume overload), motor coordination (i.e. rhythmic activities, sequences to be completed), and balance (i.e. games and recreational motor activities with emphasis on changes in center of gravity and direction, single-leg stance, and unexpected disturbances in the systems involved in balance). These components were selected for stimulation because, in neurodegenerative diseases such as Alzheimer's disease, motor impairments are noticeable and can impair functionality.

During the sessions, the main part comprised exercises involving a dual task. Participants were encouraged to simultaneously perform the proposed activity (motor stimulation) and the stimulation to concentrated attention, recognition, immediate memory, working memory, and praxis. Several types of stimulation were 
applied, such as different pictures placed on the wall and objects of different colors to be identified, memory games, counting (e.g., counting only in pairs or counting down), and simple calculations. These tasks were always combined with exercises. All participants performed the tasks together to stimulate social interaction and under the supervision of 3 to 6 physical educators or physical therapists (who had not taken part in the evaluation process).

Each session consisted of five parts (warm-up, pre-exercise stretching, exercise session, cool-down, and post-exercise stretching) using bars, Bobath balls, elastic ribbons, and medicine balls. Upper and lower limb exercises were used to stimulate the components of functional capacity and were combined with walking exercises and some circuits that included stations with the previously mentioned objects.

Often there was a progressive increase in load to follow the overload principle and promote possible gains in functional capacity each month. During each session, a different participant wore a heart rate monitor to assess the intensity of the session. Heart rate during the session remained between $60 \%$ and $80 \%$ of maximum heart rate (220 minus the participant's age in years). The program was predominantly aerobic, but in each session emphasis was placed on a specific functional component, such as strength, aerobic fitness, coordination or balance. It was not possible to assess the heart rate of every participant at each session because not enough monitors were available. Although this procedure may be considered a limitation, it seems that it is largely minimized by the fact that all the sessions were supervised and each of the participants wore the monitor in at least seven sessions throughout the training period.

The intensity of the training was low to moderate (approximately $70 \%$ of Maximal Heart Rate) to maximize the cardiorespiratory effects. However, this protocol was used on patients with neurodegenerative conditions and significant difficulties in starting any physical activity, particularly poor motor coordination, balance, and strength. Lower intensities could help patients to feel more comfortable, safe, and willing to adhere to the program.

\section{Data analysis}

Only patients with at least $75 \%$ attendance in the sessions were considered for data analysis and, for the CG, only those who attended both intervention times were considered. To characterize the demographic and clinical data of the sample population, we used descriptive statistics (mean, median, and standard deviation). A Mann-Whitney U-test was applied to verify if the groups showed significant differences in characterization data (age, schooling, and cognitive screening measured by MMSE) at baseline.

To investigate the relationship between neuropsychiatric disturbances and performance of instrumental activities,
Spearman's correlation coefficient was calculated. The continuous scores of the tests and scales pre- and post-intervention were compared using the Wilcoxon test. The effect size (ES) was calculated according to the following formula:

$\mathrm{ES}=\frac{\text { mean post-intervention }- \text { mean pre-intervention }}{\text { pre-intervention standard deviation }}$

The ES takes into account the within-group variance in performance at baseline. A high number indicates a greater therapeutic effect. For all statistical tests, a significance level of $5 \%(\mathrm{p}<0.05)$ was adopted. For analysis, we used the software SPSS, version 10.0 for Windows.

\section{Results $: \because$.}

It should be noted that during the intervention period, five participants were excluded from the EG. The primary reasons for exclusion were clinical complications, such as falls that prevented performance of the proposed protocol ( $\mathrm{n}=1$ ); unavailability of caregivers to bring participants to the sessions at the proposed time $(\mathrm{n}=3)$; progression of the disease that prevented the participant from continuing the proposed activity $(n=1)$. For the $C G$, unavailability to bring participants to the evaluation process $(n=1)$ and unwillingness to participate in the evaluation protocol $(n=1)$ were the reasons that led participants to drop out of the study.

There was no statistical difference between participants regarding age, schooling or cognitive functions given by the MMSE. There was no significant difference $(p>0.05)$ between groups in the previously mentioned variables at baseline.

The neuropsychiatric disturbances and cognitive functions had a moderate and significant correlation $(\mathrm{rho}=0.26$; $\mathrm{p}=0.02)$. Figure 1 shows the dispersion values such as frequency and suggests that the higher the cognitive impairment (lower MMSE scores) the greater the neuropsychiatric disorders (higher scores in the NPI). The correlations between cognitive functions and performance of instrumental activities

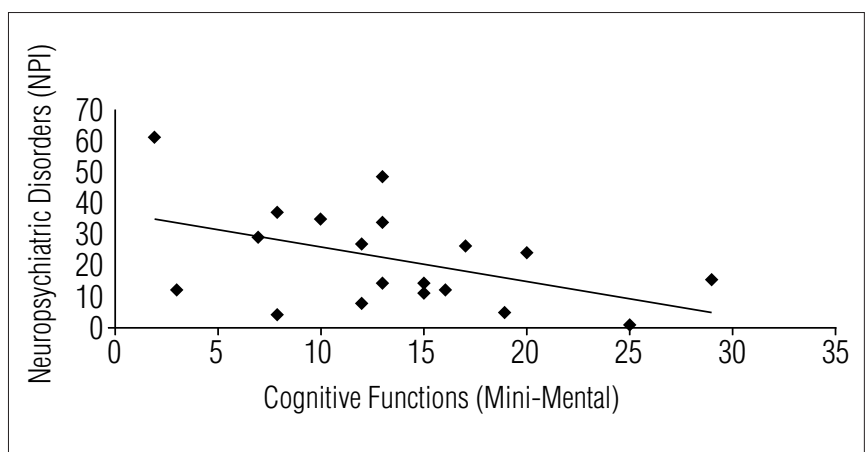

Figure 1. Relationship between neuropsychiatric disorders and cognitive functions. 
(rho=0.05; $\mathrm{p}=0.33$ ) and between neuropsychiatric disturbances and performance of instrumental activities ( $r h o=0.06 ; p=0.29$ ) were very low and non-significant.

Figure 2 shows the neuropsychiatric disturbances pre- and post-intervention for both groups (EG and CG).

The EG improved, showing a reduction in neuropsychiatric disorder scores. In contrast, the CG had lower scores for neuropsychiatric disturbances and in the total NPI values.

The Wilcoxon test was applied to analyze the effects of the motor intervention on the specific domains of the NPI, the

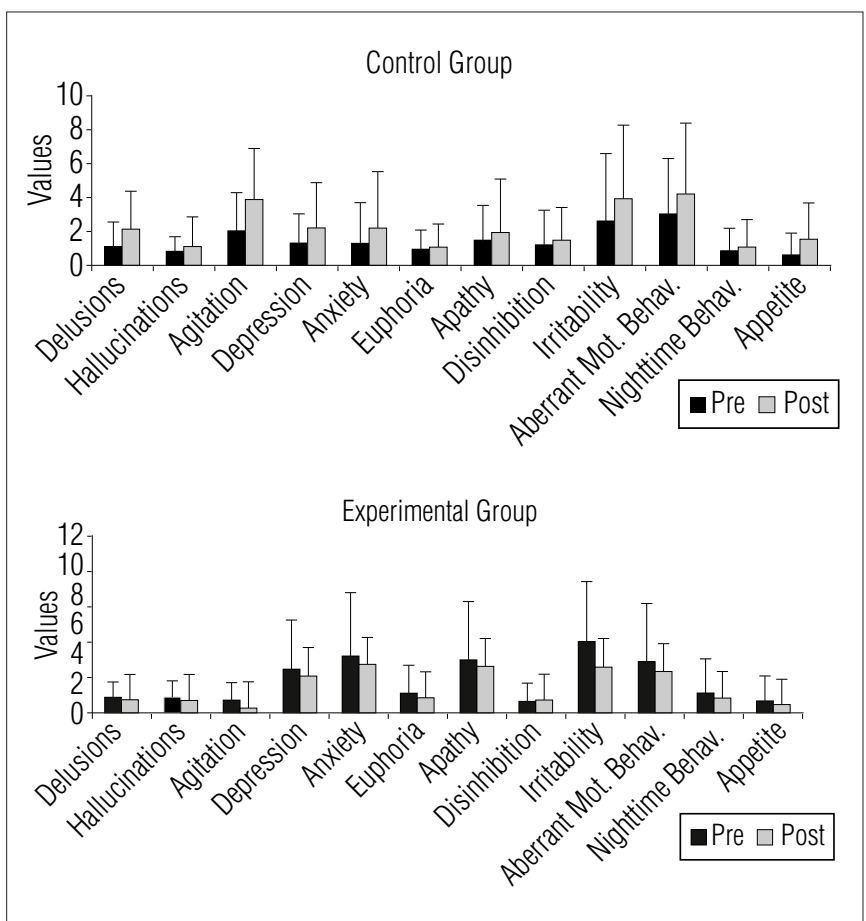

Figure 2. Neuropsychiatric disturbances in the pre- and post-intervention times for (a) control and (b) experimental group. total values of this instrument, and functional performance of both groups. The results showed a significant impairment of factors for the specific domains of NPI in the CG: Delusions $(\mathrm{Z}=-2.06 ; \mathrm{p}=0.04)$, Agitation $(\mathrm{Z}=-2.37 ; \mathrm{p}=0.02)$, and Appetite $(Z=-2.06 ; p=0.04)$. For the total NPI score, the $C G$ also showed a significant worsening $(\mathrm{Z}=-2.81 ; \mathrm{p}=-0.005)$.

For the EG, only the irritability domain showed a significant alteration $(\mathrm{Z}=-2.37 ; \mathrm{p}=0.01)$. Considering the total NPI scores, there was a significant improvement with reductions in the total score of neuropsychiatric disturbances showed by the NPI $(\mathrm{Z}=-2.09 ; \mathrm{p}=0.03)$. Table 1 shows the values of all variables evaluated as well the ES values of neuropsychiatric disturbances and performance of instrumental activities for the EG and CG.

When we compared the functional performance, we found deterioration in the CG and stability in the EG shown by the significant improvement in the Pfeffer Questionnaire score $(\mathrm{Z}=-2.67 ; \mathrm{p}=0.008)$. Figures 3 and 4 illustrate the behavior of neuropsychiatric disturbances and performance of instrumental activities for the CG and EG, respectively.

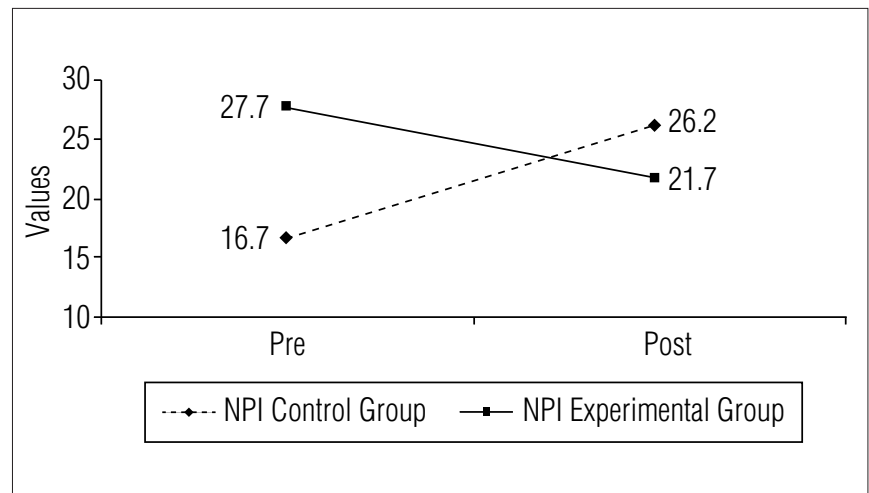

Figure 3. Scores of neuropsychiatric disturbances expressed by the NPI for the control group and the intervention group.

Table 1. Values of interaction, effect size, and significance of neuropsychiatric disturbances and instrumental activities of daily living.

\begin{tabular}{|c|c|c|c|c|c|c|}
\hline \multirow{2}{*}{ Variables } & \multicolumn{3}{|c|}{ Control Group } & \multicolumn{3}{|c|}{ Experimental Group } \\
\hline & Z & $p$ & Effect Size & Z & $p$ & Effect Size \\
\hline Delusions & -2.06 & $0.04^{*}$ & 0.7 & -0.35 & 0.72 & -0.01 \\
\hline Hallucinations & -2.37 & $0.02^{*}$ & 0.3 & -0.54 & 0.59 & -0.2 \\
\hline Agitation & -2.37 & 0.02 & 0.8 & -1.85 & 0.06 & -0.5 \\
\hline Depression & -1.84 & 0.06 & 0.6 & -0.60 & 0.54 & -0.1 \\
\hline Anxiety & -0.81 & 0.41 & 0.4 & -0.95 & 0.34 & -0.1 \\
\hline Euphoria & -1.0 & 0.31 & 0.0 & -1.63 & 0.10 & -0.2 \\
\hline Apathy & -1.07 & 0.28 & 0.3 & -0.21 & 0.83 & -0.11 \\
\hline Disinhibition & -0.44 & 0.65 & 0.2 & -1.00 & 0.31 & -0.007 \\
\hline Irritability & -1.40 & 0.16 & 0.4 & -2.37 & $0.01^{*}$ & -0.4 \\
\hline Aberrant Motor Behavior & -1.19 & 0.23 & 0.4 & -1.89 & 0.06 & -0.2 \\
\hline Nighttime Behavior & -1.41 & 0.15 & 0.1 & -0.92 & 0.35 & -0.2 \\
\hline Appetite & -2.06 & 0.04 & 0.7 & -0.81 & 0.41 & -0.2 \\
\hline NPI-Total & -2.81 & $0.005^{\star}$ & 0.7 & -2.09 & $0.03^{*}$ & -0.4 \\
\hline Instrumental Activities & -2.67 & $0.008^{*}$ & -0.02 & -0.68 & 0.49 & -0.06 \\
\hline
\end{tabular}

*Statistically significant $(p>0.05)$. 


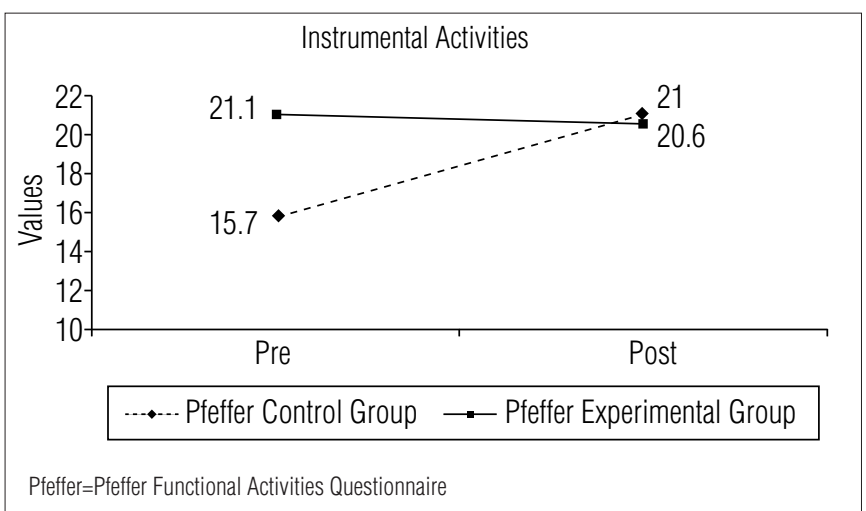

Figure 4. Scores for performance of instrumental activities of daily living expressed by the Pfeffer Functional Activities Questionnaire for control and intervention groups.

\section{Discussion $\because \because$.}

The results of the present study suggest that, for our sample of women with $\mathrm{AD}$ who performed six months of a specific multimodal exercise program, there was a significant reduction in neuropsychiatric disturbances whereas in the CG these disturbances slightly increased.

In line with our hypothesis, we found distinct benefits from physical activity. Psychopathological symptoms are commonly observed in dementia and frequently evaluated by the $\mathrm{NPI}^{24}$. These symptoms aggravate the patients' disability, with a significant negative impact on their quality of life, predicting a more rapid rate of cognitive decline and affecting their caregivers ${ }^{30}$.

In the present study, we did not classify the patients into different groups according to their CDR scores. Because of this, it was impossible to control the influence of dementia severity on NPI scores. However, the median CDR score of the groups ranged from 1.0 (1 to 3 ) to 2.0 ( 1 to 3 ), which indicated a mild to moderate level of dementia.

Experimental studies with groups of patients with dementia have shown that non-pharmacological interventions can have a positive influence on affective variables and neuropsychiatric disorders, and this has a direct reflection on the quality of life of these patients ${ }^{31-35}$. The analysis of the ES showed that the exercise program may have positive effects for this population. Differences between the groups could be attributed to the reciprocal influence of two factors: the reduction in symptoms among patients who participated in the multimodal exercise program and the increase in these symptoms in the CG.

It is known that pharmacological treatment (rivastigmine) can improve the cognitive performance and implementation of activities of daily living in the first three months of treatment. After that, there is a stabilization with small declines ${ }^{36}$ in the neurodegenerative characteristics of the disease. Taking that into consideration, participation in the intervention program seems to contribute to reduction in the progressive impairment of cognition and behavior and could alleviate patient suffering, thus helping caregivers manage their patients as shown in another study ${ }^{37}$.

The results are consistent with studies that showed clinical relevance in the positive effects of the practice of exercise combined with pharmacological treatment in $\mathrm{AD}$ patients as shown by a meta-analysis ${ }^{38}$. However, other studies analyzed by Heyn, Abreu and Ottenbacher ${ }^{16}$ in another meta-analysis did not support conclusions about the benefits of regular exercise for cognition and behavior in demented patients. A possible explanation for this could be attributed to the assessment scales, which may not have been sensitive enough to reflect these benefits ${ }^{31-33,35}$.

Other clinical trials recently verified that the exercise had a significantly good impact on behavioral disturbances and functionality in basic daily activities with a positive reduction in caregiver burden ${ }^{17,18}$. To measure psychopathological symptoms, these authors applied the Neuropsychiatric Inventory, which is the same scale used in our investigation. Despite the need to assess these symptoms, most studies carried out in this field only emphasize results considering components of cognitive function and not behavioral disturbances or functionality.

In the analysis of each disorder, we found a significant impairment in the scores for "agitation", "delusions", and "appetite". Shin et al..$^{39}$ also observed a marked prevalence of those disturbances in patients with $\mathrm{AD}$, especially agitation, which has a high prevalence in ambulatory care environments ${ }^{40,41}$. Trudeau ${ }^{42}$ found significant improvements in agitation and mood in patients who went for regular walks. It is important to remember that, according to Lyketsos ${ }^{2}$, Christofoletti et al. ${ }^{18}$, and Stella et al. ${ }^{17}$, even a slight reduction of a few points in the NPI may reflect a significant decrease in the rate of caregiver burden. Interventions that can attenuate the neuropsychiatric disturbances may have a positive impact on the quality of life of patients with dementia and their caregivers ${ }^{2}$.

Regarding the performance of instrumental activities of daily living, the EG maintained similar levels after the intervention period, showing less pronounced effects of the disease in this type of performance. The CG, however, showed greater impairment in instrumental activity scores in the post-intervention assessment when compared with pre-intervention data. The neurodegenerative process of $\mathrm{AD}$ leads to a worsening in the performance of instrumental activities of daily living and increases the burden on caregivers ${ }^{2,43}$.

Exercise interventions have been little explored in the fields of behavior and neuropsychology and in the performance of instrumental activities in patients with cognitive impairment and neuropsychiatric disorders. Baum et al. ${ }^{44}$ showed the positive effect of an exercise program on functional abilities in patients with dementia, which is in agreement with our study. Other studies did not find similar results, and their data differ in type, frequency, and intensity of the interventions ${ }^{45,46}$. 
Several forms of non-pharmacological intervention have aimed to decrease the behavioral disturbances. Management of environment, training programs for caregivers, reminiscence psychotherapy directed toward the recognition of significant past situations, sensory stimulation, strategies to enhance appropriate behaviors, and psychosocial interventions have been common ways to reduce the suffering of patients and the burden on caregivers ${ }^{47}$. However, even in geriatric institutions, few studies have investigated exercise programs focused on behavioral disturbances.

Holmberg $^{15}$ developed a regular program of walking three times a week for four months for 11 institutionalized patients with a diagnosis of dementia and observed a statistical improvement in aggression levels in those who participated in the program compared with those who did not exercise. Similarly, our sample was composed of a relatively small number of participants. However, our participants were community-dwelling patients who required additional efforts by the caregivers to transport them to the place where the exercise program was held.

Considering the maintenance of some levels even with the regular activity program, clear improvement was expected over this training period. In contrast, if changes were to occur, it would be expected that they would be in the other direction due to the progressive nature of the disease, and this outcome clearly did not occur, as shown by the sedentary participants (CG).

Dependence in activities of daily living due to dementia is associated with cognitive impairment and indicates a worsening of the dementia process. It is also a risk factor for increasing mortality ${ }^{48}$ and intensification of the neurodegenerative process $^{49}$. Dementia patients with severe impairment and requiring more assistance during the execution of activities of daily living have a risk of death three times higher than that of older adults who perform their activities normally ${ }^{50}$. According to these data, patients who postpone the onset of functional impairment and mitigate neuropsychiatric disorders tend to have a lower degree of suffering due to dementia, put less strain on caregivers, and decrease their risk of mortality ${ }^{51}$.

It is worth mentioning some methodological limitations to our study that may have weakened the findings. First, the small sample size and the design that used a non-randomized sample reduced the statistical power and capacity for generalization of our results. This study was part of a program offered to the community in which subjects participated willingly and, therefore, composed a self-selected group of highly motivated individuals. However, the sample was composed only of women, which could represent bias, considering that some variables associated with cognitive and behavioral alterations can be modulated by gender. No significant modifications in hormonal replacement therapies were reported during the course of the study.

The absence of a group of patients with severe dementia represents another limitation to the generalization of the results for the $\mathrm{AD}$ population. Because of this limitation, it was impossible to assess the effects of physical exercise on the neuropsychiatric symptoms of severe dementia or to compare the results with mild or moderate level of dementia. Nevertheless, it should be considered that it would be very difficult for a patient in advanced stages of the disease to be able to participate in such a program. Moreover, the nature of the dependence in the performance of specific activities such as exercise and the low number of professionals and volunteers to help during the training sessions made it impossible to conduct the sessions with patients in advanced stages of $\mathrm{AD}$.

In conclusion, our results suggest that six months of a multimodal exercise program is associated with a reduction in the neuropsychiatric symptoms of patients with $\mathrm{AD}$ and contributes to the attenuation of the impairment in the performance of instrumental activities of daily living in elderly women with $\mathrm{AD}$. The results must be interpreted with caution given the significant limitations of our study. However, few studies in the literature take into account the impact of exercise on behavioral and functional variables as our study did. More controlled and randomized trials should be conducted with a greater number of participants to support the present findings on the positive effects of an exercise program on neuropsychiatric disorders and performance of instrumental activities of daily living in $\mathrm{AD}$ patients.

\section{Acknowledgements $\because:$.}

The Aging and Physical Activity Laboratory, Department of Physical Education, PROCDA (Program of Functional and Cognitive Kinesiotherapy for Alzheimer's Disease) at UNESP and PROEX-UNESP.

\section{References $: \because$.}

1. Nitrini R, Caramelli P, Herrera E Jr, Bahia VS, Caixeta LF, Radanovic M, et al. Incidence of dementia in a community-dwelling Brazilian population. Alzheimer Dis Assoc Disord. 2004;18(4):241-6.

2. Lyketsos CG. Neuropsychiatric symptoms (behavioral and psychological symptoms of dementia) and the development of dementia treatments. Int Psychogeriatr. 2007;19(3):409-20.
3. Teipel SJ, Flatz WH, Heinsen H, Bokde AL, Schoenberg SO, Stöckel S, et al. Measurement of basal forebrain atrophy in Alzheimer's disease using MRI. Brain. 2005;128(Pt 11):2626-44.

4. Roach KE, Tappen RM, Kirk-Sanchez N, Williams CL, Loewenstein D. A randomized controlled trial of an activity specific exercise program for individuals with Alzheimer Disease in long-term care settings. J Geriatr Phys Ther. 2011;34(2):50-6. 
5. Scarmeas N, Luchsinger JA, Brickman AM, Cosentino S, Schupf N, Xin-Tang M, et al. Physical activity and Alzheimer disease course. Am J Geriatr Psychiatry. 2011;19(5):471-81.

6. Larson EB. Physical activity for older adults at risk for Alzheimer disease. JAMA. 2008;300(9):1077-9

7. Nitrini R, Caramelli P, Bottino CM, Damasceno BP, Brucki SM, Anghinah R, et al. [Diagnosis of Alzheimer's disease in Brazil: diagnostic criteria and auxiliary tests. Recommendations of the Scientific Department of Cognitive Neurology and Aging of the Brazilian Academy of Neurology]. Arq Neuropsiquiatr. 2005;63(3A):713-9.

8. Yaffe K. Treatment of neuropsychiatric symptoms in patients with dementia. N Engl J Med. 2007;357(14):1441-3

9. Rolland Y, Pillard F, Klapouszczak A, Reynish E, Thomas D, Andrieu S, et al. Exercise program for nursing home residents with Alzheimer's disease: a 1-year randomized, controlled trial. J Am Geriatr Soc. 2007:55(2):158-65.

10. Herrmann N, Black SE. Behavioral disturbances in dementia: will the real treatment please stand up? Neurology. 2000;55(9):1247-8.

11. Matsuda 0, Saito M. Functional competency and cognitive ability in mild Alzheimer's disease: relationship between ADL assessed by a relative/ carer-rated scale and neuropsychological performance. Int Psychogeriatr. 2005;17(2):275-88

12. Aalten P, Verhey FR, Boziki M, Bullock R, Byrne EJ, Camus V, et al. Neuropsychiatric syndromes in dementia. Results from the European Alzheimer Disease Consortium: part I. Dement Geriatr Cogn Disord. 2007;24(6):457-63

13. Eggermont L, Swaab D, Luiten P, Scherder E. Exercise, cognition and Alzheimer's disease: more is not necessarily better. Neurosci Biobehav Rev. 2006;30(4):562-75.

14. Landreville P, Bordes M, Dicaire L, Verreault R. Behavioral approaches for reducing agitation in residents of long-term-care facilities: critical review and suggestions for future research. Int Psychogeriatr. 1998;10:397-419.

15. Holmberg SK. A walking program for wanderers: volunteer training and development of an evening walker's group. Geriatr Nurs. 1997;18(4):160-5.

16. Heyn P, Abreu BC, Ottenbacher KJ. The effects of exercise training on elderly persons with cognitive impairment and dementia: a meta-analysis. Arch Phys Med Rehabil. 2004;85(10):1694-704.

17. StellaF, Canonici AP, Gobbi S, GaldurozRF, Cação Jde C, Gobbi LT. Attenuation of neuropsychiatric symptoms and caregiver burden in Alzheimer's disease by motor intervention: a controlled trial. Clinics (Sao Paulo). 2011;66(8):1353-60.

18. Christofoletti G, Oliani MM, Bucken-Gobbi LT, Gobbi S, Beinotti F, Stella F. Physical activity attenuates neuropsychiatric disturbances and caregiver burden in patients with dementia. Clinics (Sao Paulo). 2011;66(4):613-8.

19. McKhann G, Drachman D, Folstein M, Katzman R, Price D, Stadlan EM. Clinical diagnosis of Alzheimer's disease: report of the NINCDS-ADRDA Work Group under the auspices of Department of Health and Human Services Task Force on Alzheimer's Disease. Neurology. 1984;34(7):939-44.

20. American Psychiatric Association. Diagnostic and statistical manual of mental disorders. DSMIV-TR. $4^{\text {th }}$ ed. Association AP, editor. Washington: The Association; 2000.

21. Hughes CP, Berg L, Danziger WL, Coben LA, Martin RL. A new clinical scale for the staging of dementia. Br J Psychiatry. 1982;140:566-72.

22. Folstein MF, Folstein SE, McHugh PR. "Mini-mental state". A practical method for grading the cognitive state of patients for the clinician. J Psychiatr Res. 1975;12(13):189-98.

23. Brucki SM, Nitrini R, Caramelli P, Bertolucci PH, Okamoto IH. [Suggestions for utilization of the mini-mental state examination in Brazil]. Arq Neuropsiquiatr. 2003;61(3B):777-81.

24. Cummings JL. The Neuropsychiatric Inventory: assessing psychopathology in dementia patients. Neurology. 1997;48(5 Suppl 6):S10-6

25. Cummings JL, Mega M, Gray K, Rosenberg-Thompson S, Carusi DA, Gornbein J. The Neuropsychiatric Inventory: comprehensive assessment of psychopathology in dementia. Neurology. 1994;44(12):2308-14.

26. Pfeffer RI, Kurosaki TT, Harrah CH Jr, Chance JM, Filos S. Measurement of functional activities in older adults in the community. J Gerontol. 1982;37(3):323-9.

27. Laks J, Batista EM, Guilherme ER, Contino AL, Faria ME, Rodrigues CS, et al. Prevalence of cognitive and functional impairment in community-dwelling elderly: importance of evaluating activities of daily living. Arq Neuropsiquiatr. 2005;63(2A):207-12

28. Paixão Júnior $\mathrm{CM}$, Reichenheim ME. A review of functional status evaluation instruments in the elderly. Cad Saúde Pública. 2005;21(1):7-19.
29. Herrera EJr, Caramelli P, Silveira AS, Nitrini R. Epidemiologic survey of dementia in a communitydwelling Brazilian population. Alzheimer Dis Assoc Disord. 2002;16(2):103-8.

30. Bouwens SF, van Heugten CM, Aalten P, Wolfs CA, Baarends EM, van Menxel DA, et al. Relationship between measures of dementia severity and observation of daily life functioning as measured with the Assessment of Motor and Process Skills (AMPS). Dement Geriatr Cogn Disord. 2008;25(1):81-7.

31. Avila R, Bottino CMC, Carvalho IAM, Santos CB, Seral C, Miotto EC. Neuropsychological rehabilitation of memory deficits and activities of daily living in patients with Alzheimer's disease: a pilot study. Braz J Med Biol Res. 2004;37(11):1721-9.

32. Teri L, Gibbons LE, McCurry SM, Logsdon RG, Buchner DM, Barlow WE, et al. Exercise plus behavioral management in patients with Alzheimer disease: a randomized controlled trial. JAMA 2003;290(15):2015-22

33. Davis RN, Massman PJ, Doody RS. Cognitive intervention in Alzheimer disease: a randomized placebo-controlled study. Alzheimer Dis Assoc Disord. 2001;15(1):1-9.

34. Zec RF, Burkett NR. Non-pharmacological and pharmacological treatment of the cognitive and behavioral symptoms of Alzheimer disease. NeuroRehabilitation. 2008;23(5):425-38.

35. Meuleman JR, Brechue WF, Kubilis PS, Lowenthal DT. Exercise training in the debilitated aged strength and functional outcomes. Arch Phys Med Rehabil. 2000;81(3):312-8

36. Farlow M, Potkin S, Koumaras B, Veach J, Mirski D. Analysis of outcome in retrieved dropout patients in a rivastigmine vs placebo, 26-week, Alzheimer disease trial. Arch Neurol. 2003;60(6):843-8

37. Castro CM, Wilcox S, O'Sullivan P, Baumann K, King AC. An exercise program for women who are caring for relatives with dementia. Psychosom Med. 2002;64(3):458-68.

38. Forbes D, Forbes S, Morgan DG, Markle-Reid M, Wood J, Culum I. Physical activity programs for persons with dementia. Cochrane Database Syst ver. 2008;(3):CD006489.

39. Shin IS, Carter M, Masterman D, Fairbanks L, Cummings JL. Neuropsychiatric symptoms and quality of life in Alzheimer disease. Am J Geriatr Psychiatry. 2005;13(6):469-74.

40. Mega MS, Cummings JL, Fiorello T, Gornbein J. The spectrum of behavioral changes in Alzheimer's disease. Neurology. 1996;46(1):130-5.

41. Mok WY, Chu LW, Chung CP, Chan NY, Hui SL. The relationship between non-cognitive symptoms and functional impairment in Alzheimer's disease. Int J Geriatr Psychiatry. 2004;19(11):1040-6

42. Trudeau SA. Enhanced ambulation and quality of life in advanced Alzheimer's disease. J Am Geriatr Soc. 2003;51(3):429-31.

43. Selwood A, Johnston K, Katona C, Lyketsos C, Livingston G. Systematic review of the effect of psychological interventions on family caregivers of people with dementia. J Affect Disord 2007:101(1-3):75-89.

44. Baum EE, Jarjoura D, Polen AE, Faur D, Rutecki G. Effectiveness of a group exercise program in a long-term care facility: a randomized pilot trial. J Am Med Dir Assoc. 2003:4(2):74-80.

45. MacRae PG, Asplund LA, Schnelle JF, Ouslander JG, Abrahamse A, Morris C. A walking program for nursing home residents: effects on walk endurance, physical activity, mobility, and quality o life. J Am Geriatr Soc. 1996;44(2):175-80.

46. Cott CA, Dawson P, Sidani S, Wells D. The effects of a walking/talking program on communication ambulation, and functional status in residents with Alzheimer disease. Alzheimer Dis Assoc Disord. 2002;16(2):81-7.

47. Landreville P, Bédard A, Verreault R, Desrosiers J, Champoux N, Monette J, et al. Nonpharmacological interventions for aggressive behavior in older adults living in long-term care facilities. Int Psychogeriatr. 2006;18(1):47-73.

48. Landi F, Onder G, Cattel C, Gambassi G, Lattanzio F, Cesari M, et al. Functional status and clinical correlates in cognitively impaired community-living older people. J Geriatr Psychiatry Neurol. 2001;14(1):21-7

49. Gauthier S. Managing discontinuation syndrome in patients with dementia. J Psychiatry Neurosci. 2006;31(1):72.

50. Ramos LR, Simoes EJ, Albert MS. Dependence in activities of daily living and cognitive impairment strongly predicted mortality in older urban residents in Brazil: a 2-year follow-up. J Am Geriatr Soc. 2001:49(9):1168-75.

51. Jönsson L, Andreasen N, Kilander L, Soininen H, Waldemar G, Nygaard H, et al. Patient- and proxy-reported utility in Alzheimer disease using the EuroQoL. Alzheimer Dis Assoc Disord. 2006;20(11):49-55 